\title{
Influence of Vertical Coupling on the Lasing Operation of Quantum-Dash Laser
}

\author{
M. Z. M. Khan, Tien K. Ng, and Boon S. Ooi* \\ Photonics Laboratory, Computer, Electrical, and Mathematical Sciences and Engineering (CEMSE) Division, King Abdullah \\ University of Science \& Technology (KAUST), Thuwal 23955-6900, Kingdom of Saudi Arabia (KSA) \\ *corresponding author: boon.ooi@kaust.edu.
}

\begin{abstract}
The authors numerically investigated the consequence of vertical coupling among multi-stack InAs quantum dash (Qdash) laser structure on the lasing bandwidth. The developed model is based on multi-population carrierphoton rate equation and incorporates inhomogeneous broadening due to dash size or composition fluctuation, homogeneous broadening of optical gain, and the multilongitudinal photon modes. In addition, the effect of Qdash layers emitting at different wavelength, and the carrier coupling (tunneling) between adjacent stacks, are also accounted for. The results predict a direct relation between the lasing bandwidth and the barrier thickness and hence enhanced lasing bandwidth could be achieved by decoupling the Qdash layers (large barrier thickness). Moreover, the model further affirms the non-equilibrium distribution of carriers among Qdash layers in a multi-stack laser structure.
\end{abstract}

\section{INTRODUCTION}

Vertically coupled quantum dots (Qdots) and Qdashes, grown by self assembled growth technology, are widely utilized in semiconductor devices for achieving high performance. This desire to account for the coupled vertical stack Qdot/Qdash system leads to coupling of carriers (tunneling) among the layers and therefore calls for proper engineering of the laser structure design [1], [2]. Large thickness barrier/spacer layers in the multi-dot/dash layer structure reduces the vertical strain along the growth direction and hence more homogeneous dot/dash height are expected within and along the stacking layer, in addition to reducing the dislocations [3]-[5]. This is the case for the narrow band lasers, however, the opposite is true for the broadband devices. Increasing the inhomogeneity among the multi-stack layers is required to enhance the optical gain spectrum which fundamentally defines the emission bandwidth. Chirping the multi-layer device structure by varying the capping layer thickness, the nominal dash/dot monolayer thickness, and the barrier layer thickness, along different stacks, have been reported for super luminescent and laser diodes, with improved performance [6]. However, care must be exercised while varying the barrier layers among the multi-stack laser structure design as the electronic coupling enhances by reducing the barrier layer thickness.

The aim of the current work is to provide a numerical basis for the optimization of Qdash laser structural design from the viewpoint of the lasing bandwidth. Our results show that coupling effect significantly alters the lasing spectra. We find direct relation between the lasing bandwidth and the barrier layer thickness and hence enhanced lasing bandwidth could be achieved with large barrier thickness, or in other words, by minimizing the coupling effect. Moreover, we also show that the carriers are unevenly distributed among the stacking layer under equilibrium.

\section{RATE EQUATIONS}

The carrier and photon dynamics in vertically coupled Qdash lasers are calculated by solving a set of coupled rate equations. The dashes are assumed to have only one confined ground state (GS) energy level, and only electron dynamics are considered in the formulation with the hypothesis that the holes being faster follows the electrons. The reservoir of carriers is the separate-confined heterostructure $(\mathrm{SCH})$ followed by the wetting layer (WL) and the dash GS. The associated time constants are $\tau_{S W}$ (relaxation from $\mathrm{SCH}$ to $\mathrm{WL}$ ), $\tau_{W D}$ (relaxation from $\mathrm{WL}$ to Qdash), $\tau_{D W}$ (re-excitation from Qdash to WL), $\tau_{W S}$ (reexcitation from WL to $\mathrm{SCH}$ ), and $\tau_{S}, \tau_{W}, \tau_{D}$ corresponding to the recombination in $\mathrm{SCH}, \mathrm{WL}$ and Qdash layers, respectively. More details can be found elsewhere [7]. We have considered $n=1,2, \ldots N_{l y r}$ stacking layers. $N_{S C H}$ and $N_{W}$ refers to the total number of carriers in the $\mathrm{SCH}$ and $\mathrm{WL}$, while $N_{j, k}$ represents the carrier dynamics in each of the intra-dash energy levels of dash group. The subscripts $j$ and $k$ refers to the $j^{\text {th }}$ group of Qdash ensemble and its $k^{\text {th }}$ intradash energy level. The rate equations of the carrier population of these energy level are formulated in a similar manner following reference [7], taking into consideration the coupling effect. These equations are then coupled with the multimode photon rate equation:

$$
\frac{d S_{m}}{d t}=\beta \sum_{n, j, k} B\left(E_{m}-E_{j, k}^{n}\right) \frac{N_{j, k}^{n}}{\tau_{S p}}+\frac{c}{n_{a}} \sum_{n, j, k} \Gamma^{n} g_{n, m}^{j, k} S_{m}-\frac{S_{m}}{\tau_{p}}
$$

Eqn. (1) includes the spontaneous emission term, stimulated emission term and the photon loss where the photon population $S_{m}$ is proportional to the linear gain $g_{n, m}^{j, k}$ which is calculated as the sum of the contributions of the various states at the $m^{\text {th }}$ mode recombination energy $E_{m}$. The details of various terms in Eqn. 1 and other carrier rate equations are reported in literature [7]. In brief, $g_{n . m}{ }^{j, k}$ incorporates both, the homogeneous, $B\left(E_{m}-E_{j, k}^{n}\right)$ and the inhomogeneous, $G_{j, k}^{n}$, broadening. Note that the volumetric density of states (DOS) of the Qdashes, $N_{D}$, and $G_{j, k}^{n}$ includes the unique features of the dashes. The carrier relaxation and the reexcitation rates from WL to Qdash energy states are calculated according to [7] by considering the initial relaxation time $\tau_{W D O}$ (capture time when the $k^{\text {th }}$ state $j^{\text {th }}$ Qdash group is unoccupied). 
TABLE I

QDASH LASER PARAMETERS USED IN THIS SIMULATION

\begin{tabular}{c|l|c|c}
\hline \hline Parameter & \multicolumn{1}{|c|}{ Description } & Value & Unit \\
\hline \hline$L$ & Cavity length & 2000 & $\mu \mathrm{m}$ \\
\hline$d$ & Stripe width & 3 & $\mu \mathrm{m}$ \\
\hline$w_{W L}$ & Wetting layer thickness & $(1,1,1,1)$ & $\mathrm{nm}$ \\
\hline$w_{D H}$ & Qdash width & $(3.2,2.9,2.6,2.3)$ & $\mathrm{nm}$ \\
\hline$h_{D H}$ & Qdash height & 4 & $\mathrm{~nm}$ \\
\hline$N_{l v r}$ & Number of Qdash layers & - \\
\hline$A_{e f f}$ & Qdash effective crossection area & $(8.0,7.2,6.5,5.7) \times 1 \mathrm{e}-11$ & $\mathrm{~cm}^{2}$ \\
\hline$\Gamma$ & Confinement factor & $(0.01,0.01,0.01,0.01)$ & - \\
\hline$N_{D}$ & Qdash density of states & $1 \mathrm{e} 18$ & $\mathrm{~cm}^{-3}$ \\
\hline$E_{C V}$ & Central transition energy & $(743,763,783,803)$ & $\mathrm{meV}$ \\
\hline$E_{W L}$ & WL ground state energy & 890 & $\mathrm{meV}$ \\
\hline \hline
\end{tabular}

\section{RESULTS AND DISCUSSION}

The model has been solved in time domain with the fourth order Runge-Kutta method to obtain the steady state solutions. The InAs/InP Qdash laser utilized in the current work is taken from [2] and the parameters used in the simulation are summarized in Table. 1 [2], [7]. The room temperature calculated lasing spectra at increasing current injection are shown in Fig. 1(a). The localized inhomogeneous broadening of the layers are fixed at $(30,30,25,25) \mathrm{meV}$. A progressive increase in the $3 \mathrm{~dB}$ bandwidth (calculated at full width at half maximum, FWHM) is evident from the figure. This is attributed to the initial lasing from dash groups with intermediate average height (since height basically defines the GS energy level) once the total device loss is compensated, and gradual contribution from the smaller height dash groups, as a result of band filling effect, thus, broadening the lasing spectra towards the shorter wavelength. In addition, the broadening at the longer wavelength indicates that the larger height dash groups also starts lasing once they reach the condition of stimulated emission. Note that there is always competition between the small and large dash groups since the photon reabsorption process cannot be ignored, but, with increasing injection, it is possible for both the groups to lase concurrently, thus enhancing the lasing spectra. Since, we have considered a barrier thickness of $30 \mathrm{~nm}$ in the above case, the migration of carrier across the stacks is restricted (tunneling time $\sim 2 \tau_{W D O}$ ) which eventually decouples the layers. Therefore, the inhomogeneity of each dash layers persists and the carriers experience these inhomogeneous broadening separately. This enhances the overall inhomogeneity of the decoupled system, leading to large emission broadening. However, this is not the case with smaller barrier thickness structures as depicted in Fig. 1(b) where the FWHM of the lasing emission is plotted against the barrier thickness (identical among all the stacking layers) at low and high current injection. Coupling among the stacking layers enhances with decreasing barrier thickness which reduces the lasing bandwidth $(\sim 54 \mathrm{~nm}$ with $30 \mathrm{~nm}$ barrier compared to $\sim 31 \mathrm{~nm}$ with $15 \mathrm{~nm}$ barrier, at $5.0 \mathrm{I}_{t h}$ ). This is because, the group of dashes that are contributing to the lasing and serving as a fast sink for excess carriers, could get the carriers from other dash groups of adjacent stacks, with low recombination rate, through coupling (tunneling time $\sim 0.6$ $\left.\tau_{W D 0}\right)$. Therefore, the carriers in the coupled system experiences delocalized inhomogeneous broadening which eventually reduces the lasing bandwidth. From Fig. 1(b), it can be seen that the optimum barrier thickness required to maintain the decoupling effect is $\sim 30$ to $50 \mathrm{~nm}$. Thus, this is the suggested barrier thickness range for the optimum broadband laser structure design. Moreover, we have also noticed that the carrier population in the different dash layers at equilibrium ( steady state) is uneven with the dash layer near to the p-
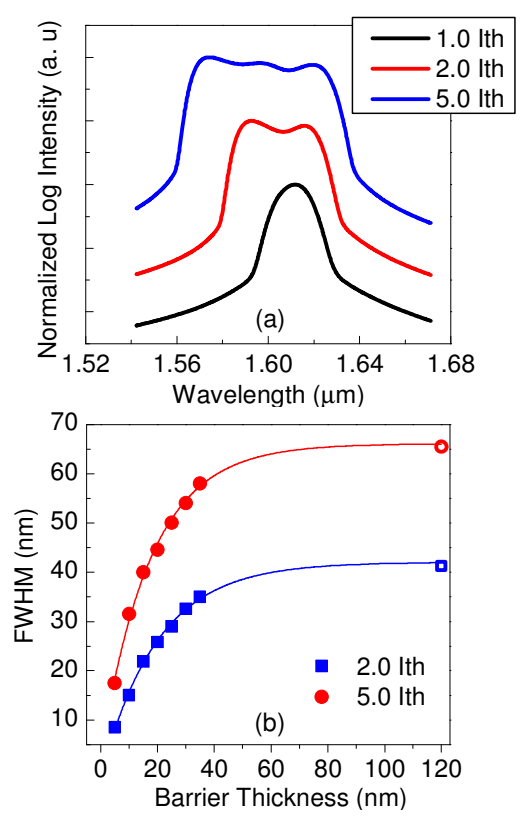

Fig. 1. Calculated (a) The progressive lasing spectra of the Qdash laser structure with $30 \mathrm{~nm}$ barrier with current injection, and (b) FWHM (solid symbols) of the Qdash laser structure as a function of barrier layer thickness, and at two different current. The hollow symbols are the calculated FWHM values with infinite barrier thickness. The solid lines are the exponential fit to the calculated data symbols.

cladding being accompanied with more carriers than the dash layer near to n-cladding regardless identical dash DOS in each stack. Our work further reconfirms the non-equilibrium distribution of carriers among the stacking layers as a result of active region inhomogeneity.

\section{CONCLUSION}

The paper investigated the effect of barrier thickness on the lasing bandwidth of InAs/InP Qdash lasers. Our model reveals that the lasing bandwidth decreases with decrease in barrier thickness as a result of enhanced carrier coupling among the stacking layers.

\section{ACKNOWLEDGMENT}

The work is supported by KAUST- University of Michigan's AEA 2010 Grant.

\section{REFERENCES}

[1] F. Lelarge, et al., "Recent advances on InAs/InP quantum dash based semiconductor lasers and optical amplifiers operating at $1.55 \mathrm{~m}, "$ IEEE Journal of Selected Topics in Quantum Electronics, vol. 13, pp. 111-124, 2007.

[2] H. S. Djie, et al., "Ultrabroad stimulated emission from quantum-dash laser", Appl. Phys. Lett., vol. 91, pp. 111116, 2007.

[3] B. Shi and Y. Xie, "Influence of coupling effect in the operation of vertically coupled quantum-dot lasers," ", Appl. Phys. Lett., vol. 82, pp. 4788-4790, 2003

[4] K. Nishi, et al., "A narrow photoluminescence linewidth of $21 \mathrm{meV}$ at $1.35 \mu \mathrm{m}$ from strain-reduced InAs quantum dots covered by In $0.2 \mathrm{Ga} 0.8$ As grown on GaAs substrates", Appl. Phys. Lett., vol. 74, pp. 1111-1113, 1999.

[5] O. Qasaimeh, "Vertical coupling in multiple stacks quantum-dot semiconductor optical amplifiers," Journal of Physics D: Applied Physics, vol. 42, p. 234001, 2009.

[6] P. Bardella, et al., "Modeling of broadband chirped quantum-dot superluminescent diodes," IEEE Journal of Selected Topics in Quantum Electronics, vol. 15, pp. 785-791, 2009.

[7] M. Z. M. Khan, et al., "Spectral Analysis of Quantum Dash Lasers: Effect of Inhomogeneous Broadening of the Active Gain Region," IEEE Journal of Quantum Electronics, vol. 48, pp. 608-615, 2012. 